\title{
Analysis of pollutant entrainment From localised sources in a street network
}

Article

Accepted Version

Cezana, F. C., Goulart, E. V., Reis Jr, N. C. and Coceal, O. ORCID: https://orcid.org/0000-0003-0705-6755 (2021)

Analysis of pollutant entrainment From localised sources in a street network. Boundary-Layer Meteorology, 179. pp. 241258. ISSN 0006-8314 doi: https://doi.org/10.1007/s10546-02000598-7 Available at https://centaur.reading.ac.uk/94902/

It is advisable to refer to the publisher's version if you intend to cite from the work. See Guidance on citing.

To link to this article DOI: http://dx.doi.org/10.1007/s10546-020-00598-7

Publisher: Springer

All outputs in CentAUR are protected by Intellectual Property Rights law, including copyright law. Copyright and IPR is retained by the creators or other copyright holders. Terms and conditions for use of this material are defined in the End User Agreement.

\section{www.reading.ac.uk/centaur}

\section{CentAUR}

Central Archive at the University of Reading 
Reading's research outputs online 


\title{
Analysis of Pollutant Entrainment From Localised Sources in a Street Network
}

\author{
3 FC Cezana • EV Goulart • NC Reis Jr • \\ 4 O Coceal
}

5

6 Received: DD Month YEAR / Accepted: DD Month YEAR

7 Abstract The propagation of a pollutant emitted from localised sources both within and above a regular street network is studied by analysing data from direct numerical simulations of passive scalar dispersion. Two wind directions are considered, corresponding to aligned and oblique flow with respect to the street axes. Particular attention is paid to the role of entrainment of the scalar into the urban canopy from an elevated source and re-entrainment of material originally released further upstream from a ground source. The variation of concentration differences and vertical fluxes between the streets and the air above as a function of distance reveals important differences between the rate of lateral and vertical mixing for the two sources. Detrainment and entrainment need a longer fetch to equilibrate for the elevated source than for the ground source. There are large differences between the advection and detrainment velocities for the aligned and oblique cases, so that a change in wind direction could affect ventilation efficiency considerably. Time scales associated with different dispersion processes are computed and the time of first appearance of

FC Cezana

Federal University of Espirito Santo, Vitoria, Brazil

EV Goulart

Federal University of Espirito Santo, Vitoria, Brazil

Tel.: +55-27-40092177

Fax: +55-27-40092148

E-mail: elisa.goulart@ufes.br

NC Reis Jr

Federal University of Espirito Santo, Vitoria, Brazil

O Coceal

National Centre for Atmospheric Science (NCAS), Department of Meteorology, University of Reading, PO Box 243, Reading, RG6 6BB, UK 
the scalar from the onset of release in different streets is mapped. It is shown that re-entrainment can provide a shortcut dispersion pathway for reaching certain parts of the network. This is particularly striking in the case of oblique flow, when material can be transferred by entrainment up to twice as fast as it could by advection. Taken together, these results highlight the overall message that vertical exchange is a two-way process and that entrainment needs to be considered in the context of emergency-response as well as urban ventilation.

Keywords Air pollution · Direct numerical simulation · Pollutant entrainment $\cdot$ Street network · Urban dispersion

\section{Introduction}

Cities are the world's major economic, social and geographical centres, concentrating most of the global investments and resources. Consequently, the number of people living in urban spaces is growing. However, demographic growth has been accompanied by several problems, among them atmospheric pollution. Thus, it is expected that human exposure to hazardous substances is higher, especially in areas where the density of population and traffic are relatively high. The urban morphology can either dilute or increase the concentration of pollutants at pedestrian level, depending on complex local dispersion processes and buiding geometry. Therefore, understanding the transport and dispersion of pollutants in populated areas is an important aspect of air quality management and mitigation strategies. Due to the complexity of the urban environment and the interplay of different flow processes several aspects of urban dispersion remain little-understood (Britter and Hanna 2003; Soulhac et al. 2009; Belcher et al. 2012). One such process is entrainment of pollutants into the urban canopy, either from outside sources or from material originally released within the canopy further upstream (re-entrainment). This process is an important determinant of the general issue of urban ventilation or urban breathability (Neophytou and Britter 2005), which is gaining greater recognition as a critical aspect of urban sustainability (Peng et al. (2020)).

The concept of breathability has been linked to that of the vertical exchange velocity (Neophytou and Britter 2005). This link has been invoked in numerous subsequent studies (e.g. Panagiotou et al. (2013); Chen et al. (2017); Shen et al. (2017)). For instance, Chen et al. (2017) investigated how the building height influences city breathability using wind tunnel experiments and computational fluid dynamics (standard $k-\epsilon$ ) modelling in a medium-density and a compact-density model. The authors investigated the in-canopy horizontal velocity and the exchange velocity at the top of the canopy which they related to city breathability. They found that for medium-density models the building height variations increase the exchange velocity of taller buildings, but reduces that of lower buildings. The compact-density urban model had weaker in-canopy horizontal velocity and vertical turbulent exchange velocity than the medium-density model. Shen et al. (2017) conducted a study of dispersion 
of a passive scalar in the turbulent flow over arrays of cubes under neutral and stable stratification using a large-eddy simulation. The study was designed to investigate the effect of the plan area density on the flow and dispersion. They found that in general, as the plan area density increases the in-canopy concentration is higher. For a skimming flow, the authors showed that for a stable case the vertical transportation of scalar is weaker and therefore the concentration is higher within the canopy compared to the neutral case. For neutral conditions, the advection scalar flux within the canopy reduces with distance from the source as the plan area density increases. The authors suggested that this reduction is due to the enhancement of the vertical scalar transfer. For stable conditions, advection scalar flux within the canopy has a slower decrease with distance from the source because the scalar transfer at canopy top is relatively weaker. Chen et al. (2017) and Shen et al. (2017) are merely two examples of many recent studies that highlight the importance of characterising vertical exchange for understanding urban ventilation. However, vertical exchange is a two-way process; while there is an abundance of experimental and numerical work on detrainment of pollutants out of the urban canopy top, there has been very little work on entrainment into the canopy.

Basic understanding of entrainment is needed for several reasons, both practical and fundamental. First, it is relevant for source attribution assessments and emission reduction measures. For example, if significant amounts of pollutants released elsewhere are entrained in already polluted areas, then the effectiveness of clean air zones for controlling local emissions may need to be re-examined. Secondly, accidental releases from industrial areas may spread toxic fumes and particulates over neighbouring residential areas or city centres via entrainment. Such was the case, for instance, during a major fire incident at the Buncefiled oil depot north of London, UK, during December 2005. Enormous quantities of PM10 were released, equivalent to 6 per cent of the annual UK emission, which led to the closure of hundreds of schools and public places and home evacuations over two days (Targa et al. 2006). Thirdly, even under normal conditions the air above an urban area will have a different concentration of pollutants and possibly different chemical composition from that within streets. For example larger ozone concentratons may be advected from rural surroundings. Hence, entrainment may alter the types and rates of chemical reactions in streets (Harrison 2018). Fourthly, dispersion models need to include the effect of entrainment, e.g. Belcher et al. (2015), Goulart et al. (2018) and Hertwig et al. (2018) showed that it becomes important within a few streets downstream of a release and that the performance of a street network dispersion model is substantially improved by taking it into account.

Aside from the insights gained from the modelling studies of Belcher et al. (2015), Goulart et al. (2018) and Hertwig et al. (2018), there is little in the published literature focusing on entrainment and re-entrainment in urban canopies. Garbero et al. (2010) performed a series of wind-tunnel experiments to study different transfer processes in a street network, including channelling along streets, mixing in intersections and vertical exchange between the streets and the overlying flow. However, they did not consider entrainment explicitly. 
Belcher et al. (2012) refers to unpublished material (DAPPLE 2011) which showed some evidence of the effect of entrainment and re-entrainment from elevated and ground sources respectively based on wind-tunnel measurements pertaining to a site in central London. The results showed that entrainment caused a widening of the initially narrow plume from a localised elevated source once the material entered the street network, where the plume angle spanned $90^{\circ}$ a sector. For a street level emission, the plume initially dispersed within a $90^{\circ}$ sector as a result of the local building geometry and was thereafter confined to a slightly narrower sector. Carpentieri et al. (2012) and Carpentieri et al. (2018) performed detailed wind-tunnel measurements of pollutant fluxes and quantified the mean and turbulent components of horizontal and vertical fluxes. Hertwig et al. (2018) related these wind-tunnel results to the role of entrainment. Given the paucity of experimental data on entrainment the use of simulated data should be considered. Direct numerical simulation (DNS) and large-eddy simulation (LES) are well-established tools for performing fundamental studies of flow and dispersion (Belcher et al. 2012). Indeed the use of such data is sometimes preferable to the direct use of experimental measurements, provided they are validated first. Advantages of such simulations include: they can be performed under controlled conditions, can be designed to focus on particular processes and produce data at much higher spatial resolution than is typically possible experimentally. In particular, DNS is different from other modelling in that it is a direct solution of the NavierStokes equations without any modelling assumptions (Moin and Mahesh 1998; Pope 2000). The only errors in DNS are due to finite discretization and hence error margins in DNS are typically lower than experimental ones. DNS simulations over urban-like geometry have yielded results in excellent agreement with carefully-conducted wind-tunnel experiments (Coceal et al. 2006, 2007).

In relation to the under-explored subject of entrainment, key basic questions that need to be addressed include: (i) How much material is entrained, and how quickly? (ii) When and where is entrainment most important? (iii) What controls it in an urban canopy? This paper presents a preliminary study of these questions by analysing data from direct numerical simulations (DNS) of passive scalar dispersion over an idealised street network. The data used and the simulations that generated them are presented in section 2. In view of the focus on entrainment and re-entrainment, the dispersion characteristics from an elevated source are compared against those from a ground source (section 3). The development in space and in time of the concentration through the network is characterised and interpreted in terms of underlying dispersion mechanisms and time scales linked with different processes (section 4). We summarise the main findings and highlight their novelty in section 5 .

\section{Numerical Modelling and Data}

The data used in this study is based on direct numerical simulation (DNS) of turbulent flow over arrays of cubes, which represent an idealised urban 
area. The numerical methods are described in Coceal et al. (2006) and Coceal et al. (2007). In brief, the Navier-Stokes equation was discretized using a second-order central finite difference scheme in space and a second-order Adams Bashforth scheme in time, based on the pressure correction method. The Poisson equation for pressure was solved using a multigrid method. The code was parallelized using Message Passive Interface (MPI).

The DNS runs that generated the datasets analysed here are described in Branford et al. (2011), and they additionally solved the passive scalar equation coupled to the computed velocity field, with an ensemble of localised sources as described below. Detailed descriptions of the numerical methods, tests and validation of flow statistics against wind-tunnel data are given in Coceal et al. (2006) and Coceal et al. (2007) and corresponding details and validation of concentration statistics against water-channel data are reported in Branford et al. (2011). Essential details of the simulations are summarised in the following.

A plan view of the domain setup is shown in Figure 1 and involves a regular array of cubical obstacles of height $h$. The domain size was $16 h \times 16 h$ in the horizontal and $8 h$ in the vertical. In the present work we analyse data from two runs in which the wind direction is at $0^{\circ}$ and $45^{\circ}$ to the cube array. The simulations were conducted under conditions of neutral stability and fully rough turbulent flow. The Reynolds number based on the velocity magnitude at the top of the domain and the cube height was typically between 4750 and 7000 . The flow was maintained by a height-independent pressure gradient of magnitude $u_{\tau}^{2} / H$, where $u_{\tau}$ is the total wall friction velocity and $H$ is the domain height. The imposed boundary conditions were periodic in the horizontal directions, free-slip at the domain top and no-slip on the bottom and all cube surfaces.

A non-dimensional time scale characterizing the turnover time of eddies shed from the cubes can be defined as $T=h / u_{\tau}$. The simulations were run with a time step of $0.00025 T$. Each run was spun up for a duration of approximately $200 T$ to allow fully developed turbulence conditions. After this spin-up time, passive scalar was switched on and released at a steady rate $q=0.0574684$ (mass per unit volume per unit time, in units chosen such that $h=1, u_{\tau}=1$ and air density $\rho=1$ ) thereafter from an ensemble of sources located close to the ground (at $z=0.0625 h$ ) within the array at $z=2 h$ above the array; the source locations are indicated in Fig. 1. A sponge layer was applied at the boundary of the domain to prevent the scalar from re-entering the domain. At the top of the domain the scalar was allowed to escape.

For each run the sources were placed in equivalent locations so that they formed an ensemble of equivalent simultaneous releases. Statistics were then collected and averages computed over an interval of approximately 100T. Averaging over an ensemble of releases helped in reducing the overall computational cost as it is equivalent to increasing the duration of the time series of one individual release for the same flow simulation. The ensemble-averaging was done by shifting the origin of the coordinate system for each source as follow: for the $0^{\circ}$ run, such that the effective source location in each case is at $(3.5 h, 6.5 h)$ 
(a)

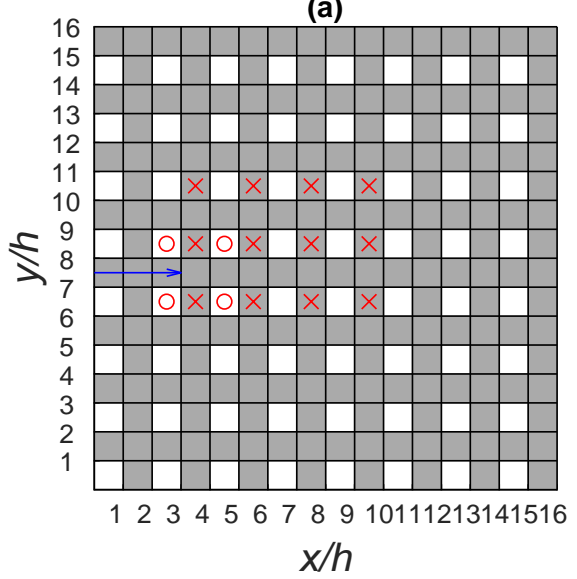

(b)

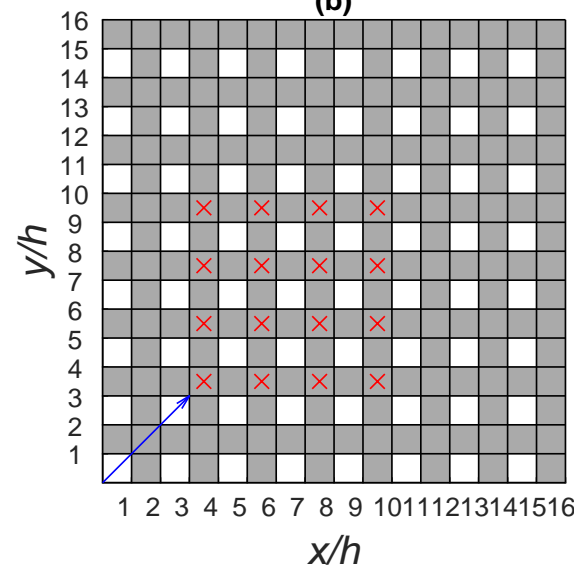

Fig. 1 Plan view of the computational domain in the DNS for a forcing direction of (a) $0^{\circ}$ and (b) $45^{\circ}$. White squares denote building positions and grey areas denote the air space between them. Locations of ground sources at $z=0.0625 h$ and elevated sources at $z=2 h$ are denoted by red crosses and circles respectively. Blue arrows indicate forcing wind directions.

for the ground source and at $(2.5 h, 6.5 h)$ for the elevated source; for the $45^{\circ}$ run, the corresponding shifted location is at $(3.5 h, 3.5 h)$. The result of this averaging is to produce concentration fields and time-series corresponding to a single release at the given effective source location in each case.

For the $0^{\circ}$ case, we shall refer to obstructed regions between buildings as 'canyons' and to other unobstructed streets as 'channels', of which there are two types: those between cubes and those between canyons. We expect different flow patterns and hence different dispersion behaviours among these three types of regions. For the $45^{\circ}$ case there are only two types of regions: 'intersections', which are directly linked to 'streets' on all four sides; the streets themselves are between cubes on either side and are linked to intersections at their ends. Hence, one might expect them to share characteristics of both the canyons and channels of the $0^{\circ}$ case.

\section{Comparison Between Dispersion From an Elevated Source and a Ground Source}

A source located above a street network gives rise to characteristically different dispersion patterns than a source within the network. In this Section we describe these differences and explain them in terms of underlying dispersion processes. 


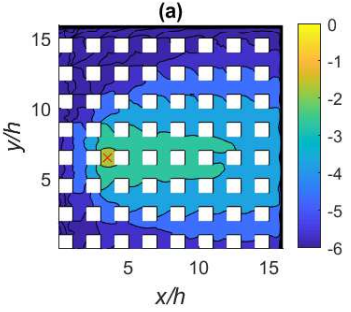

(c)

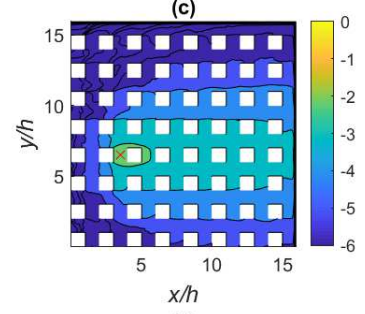

(e)

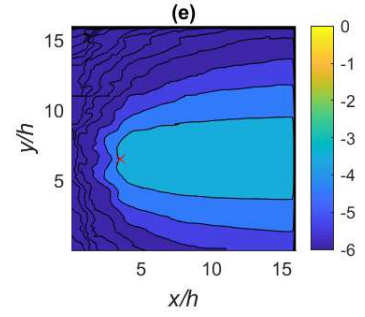

(b)

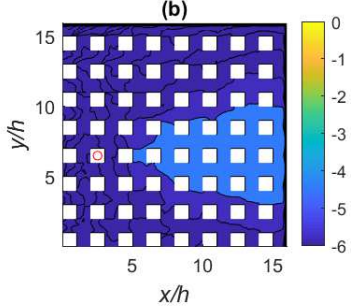

(d)
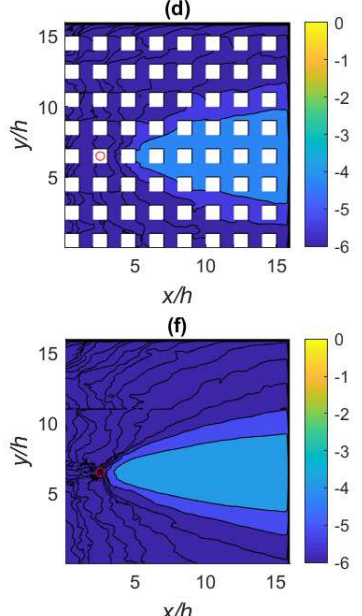

$x / h$

Fig. 2 Contours of ensemble-averaged mean concentration at three different heights (a) and (b) at $z=0.5 h,(\mathrm{c})$ and (d) at $z=h,(\mathrm{e})$ and (f) at $z=1.5 h$, for the ground source (left panels) and elevated source (right panels) for the $0^{\circ}$ run. The colour bars and concentration contours correspond to the common logarithm $\left(\log _{10}\right)$ of the concentrations. Locations of ground source at $z=0.0625 h$ and elevated source at $z=2 h$ are denoted by red crosses and circles respectively.

\subsection{Mean Concentration Pattern}

Fig. 2 shows contours of the ensemble-averaged mean concentration at three different heights $(z=0.5 h, z=h$ and $z=1.5 h)$ for the ground source (left panels) and the elevated source (right panels) for the $0^{\circ}$ run. Not surprisingly, there are several qualitative and quantitative differences in the dispersion patterns from these two release locations. For the ground source by far the highest concentration is found in the canyon where the source is located, indicating trapping of the scalar in the wake of the building immediately behind it. This persists up to the building height $z=h$, where the region of high concentration is seen to entend into the wake of the next building downstream. A nested series of roughly oblong-shaped contours enclose regions of comparable concentration within the array. Above the array, at $z=1.5 h$, these regions open up slightly downstream. In comparison, the corresponding concentration pattern at the same height from the elevated source located at $z=2 h$ is more triangular and similar to a Gaussian plume shape. The difference can be 
understood by thinking of the time-averaged pattern from the ground source as resulting from a much more diffuse effective source in the canopy underneath, once a dynamic equilibrium has been established (Goulart et al. 2018). As a consequence, the plume from the ground source differs markedly from a Gaussian shape both within and above the array, although it approximates a Gaussian far from the source (Coceal et al. 2014; Belcher et al. 2015).

Fig. $2 \mathrm{~b}$ and $2 \mathrm{~d}$ show that entrainment from the elevated source into the urban canopy occurs fairly rapidly, within about two building heights downstream of the source. The resulting plume of entrained material retains a triangular shape within the array, and widens slightly. Comparison of Fig. $2 \mathrm{~b}$ and Fig. 2f shows that the rate at which the plume widens once it is in the array is quicker than that of the material above. This is reminiscent of the wind-tunnel results from DAPPLE (2011) alluded to in the Introduction.

The entrainment of material into the canopy from the elevated source, and re-entrainment from the ground source can be seen more clearly in the contour plots in vertical planes shown in Fig. 3. For the ground source, initial rapid detrainment out of the canyon where the source is located is followed by re-entrainment over the next few canyons. Further on, the balance of these two processes results in a rapid approach to equilibrium, where the concentration within and above the array are eventually equalised. Similar results were reported by Goulart et al. (2018) and Hertwig et al. (2018). Fig. 3a and 3c show that this happens sooner over the row of buildings than over the open channels. The opposite appears to be the case for the elevated source. Here the approach to equilibrium happens quicker over the channels, and it is more gradual compared to the ground source. Despite the fairly rapid entrainment, the concentration within the canopy does not approach that above as quickly as for the ground source.

\subsection{Concentration Differences Within and Above the Street Network}

To analyse the approach to equilibrium more quantitatively, Fig. 4 plots the ensemble-averaged mean concentration at two heights $z=0.5 h$ and $z=1.5 h$ as a function of distance at lateral locations $y=6.5 h$ and $y=7.5 h$ for the ground source (Fig. 4a) and the elevated source (Fig. 4b). These locations were chosen to correspond to the unobstructed streets along which most of the scalar is channelled, due to their vicinity to the source location. Note that the concentration at $z=0.5 h$ within the canyon in which the source is located (at $x=3.5 h$ ) is off the scale of the plot, at $\approx 0.058$, nearly an order of magnitude larger than the value within the immediately adjacent channel at $z=0.5 h$ (which is $\approx 0.0064$ ) and more than two orders of magnitude larger than the concentration immediately above at $z=1.5 h$.

Fig. 4a shows that the lateral concentration differences in the array are generally small for the ground source, except for the canyon in which the source is located compared to the adjacent street in the channel, as noted above. There are somewhat larger lateral differences in the concentrations 

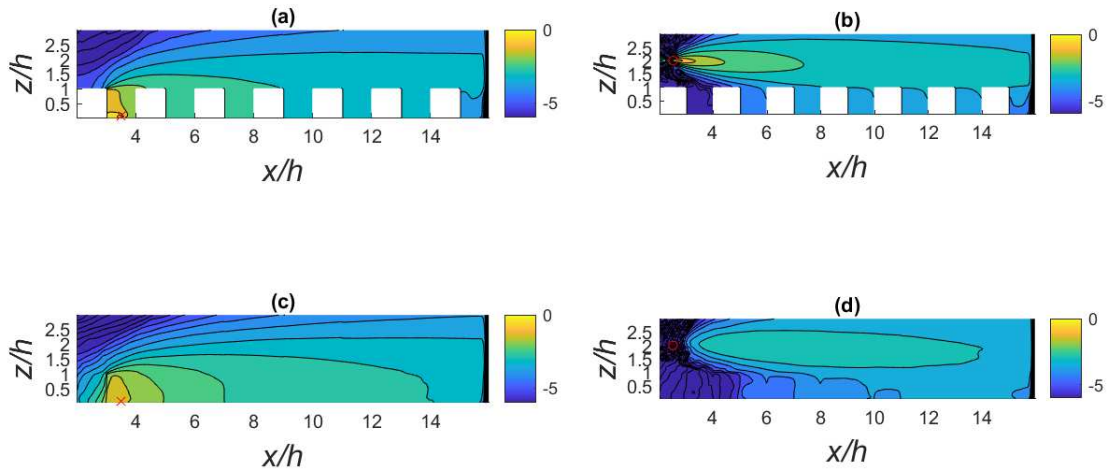

Fig. 3 Contours of ensemble-averaged mean concentration in two vertical planes (a) and (b) at $y=6.5 h,(\mathrm{c})$ and (d) $y=7.5 h$ for the ground source (left panels) and elevated source (right panels) for the $0^{\circ}$ run. The colour bars and concentration contours correspond to the common logarithm $\left(\log _{10}\right)$ of the concentrations. Locations of ground source at $z=0.0625 \mathrm{~h}$ and elevated source at $z=2 h$ are denoted by red crosses and circles respectively.

above the array, but these differences quickly decrease, and indeed all the four concentration profiles nearly converge by the time the end of the array is reached. For the elevated source, Fig. 4b shows that the lateral differences are again relatively small within the array but are now considerably larger above, due to the narrower plume from this source. Fig. 4c plots the absolute value of the concentration difference between each street and above it as a fraction of the sum of those concentrations for each source from the data shown in Fig. $4 \mathrm{a}$ and Fig. 4b. These plots reinforce the observations made in the previous section on the relative rate of approach to equilibrium.

The effect of lateral dispersion is particularly important for oblique flow directions, when it is enhanced by a topological mechanism, namely the diverging of mean streamlines around buildings (Belcher et al. 2015). This is illustrated in Fig. 5, which shows the ensemble-averaged and box-averaged mean concentration in each street resulting from a ground source release at $(3.5 h, 3.5 h)$. The enhanced lateral dispersion coupled with strong initial detrainment results in a rapid decrease in the centreline concentration with distance from the source, so that the concentrations within and above match after a distance of only about $4 h$ downstream of the source. Note again that the concentration in the street in which the source is located (at $x=3.5 h$ ) is off the scale of the plot, at $\approx 0.0361$.

\subsection{Detrainment and Entrainment Scalar Fluxes Across the Roof Level}

The magnitude of the detrainment and entrainment across the canopy top can be quantified by computing the vertical flux (mean plus turbulent), decom- 

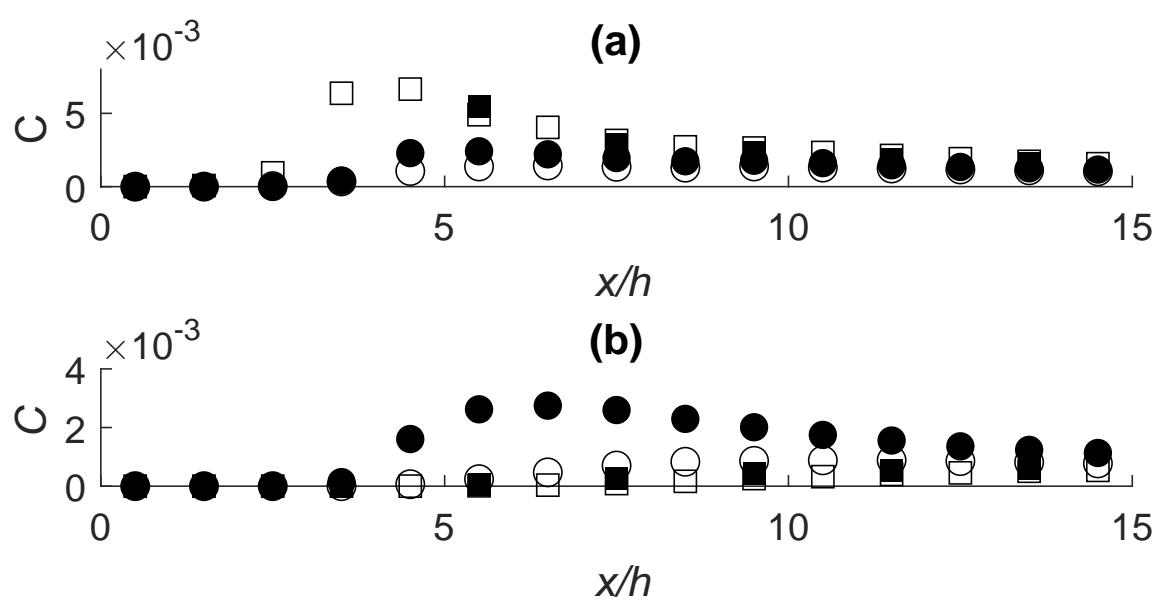

(c)

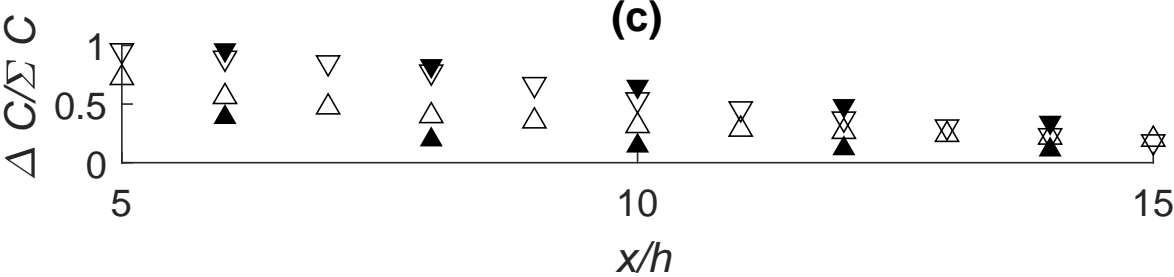

Fig. 4 Magnitudes of ensemble-averaged mean concentration at different distances along the forcing direction for (a) the ground source, (b) the elevated source, for the $0^{\circ}$ run. Squares denote locations within the array at $z=0.5 h$ and circles locations above the array at $z=1.5 \mathrm{~h}$. Filled symbols correspond to lateral locations at $y=6.5 \mathrm{~h}$ and empty symbols at $y=7.5 h$ respectively. (c) Ratio of absolute difference to sum of concentrations within and above each street, $\Delta C / \Sigma C$, for the ground source (arrows up) and elevated source (arrows down). Filled and empty symbols as for (a) and (b).

(a)

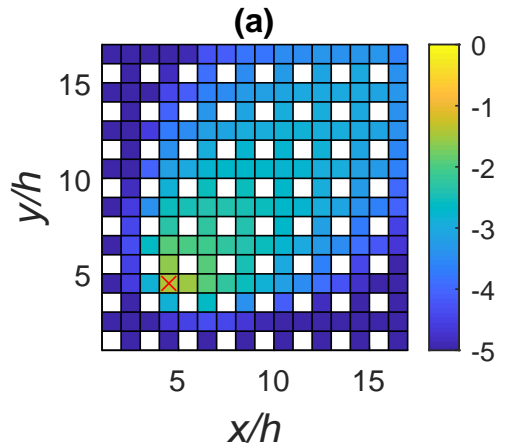

(b)

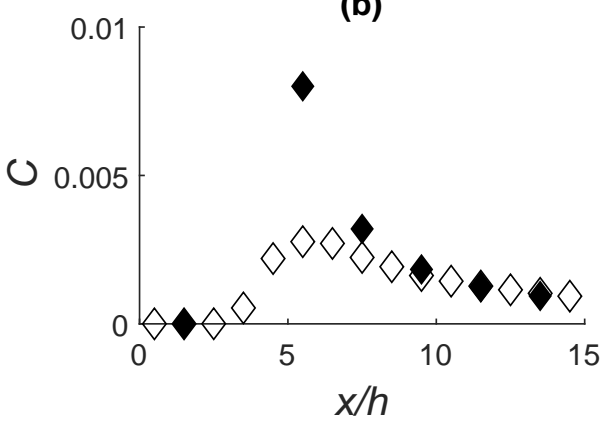

Fig. 5 (a) Map of ensemble- and box-averaged mean concentration and (b) magnitudes of ensemble-averaged mean concentration at different distances along the plume centreline for the $45^{\circ}$ run. The colour bars correspond to the common logarithm $\left(\log _{10}\right)$ of the concentrations. Filled symbols indicate concentrations within the array at $z=0.5 h$ and empty symbols concentrations above the array at $z=1.5 h$. Location of ground source at $z=0.0625 h$ is denoted by a red cross in (a). 
(a)

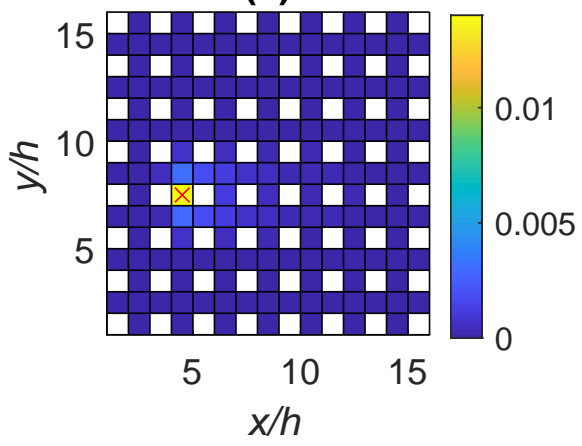

(c)

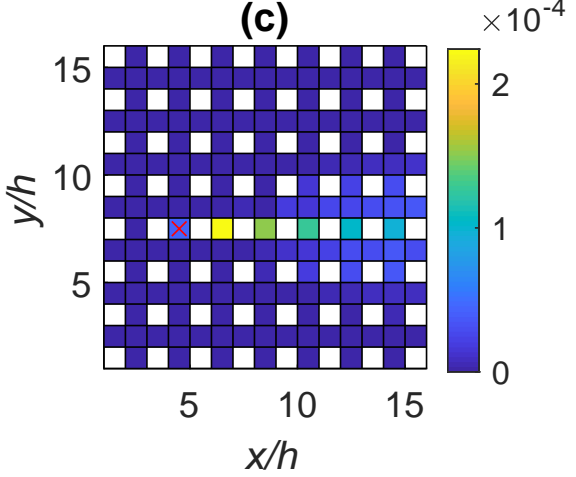

(b)

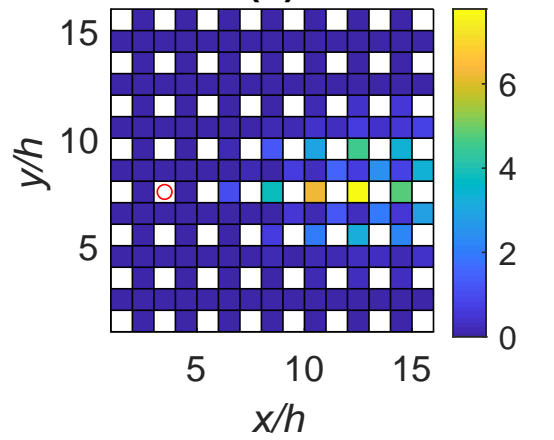

(d)

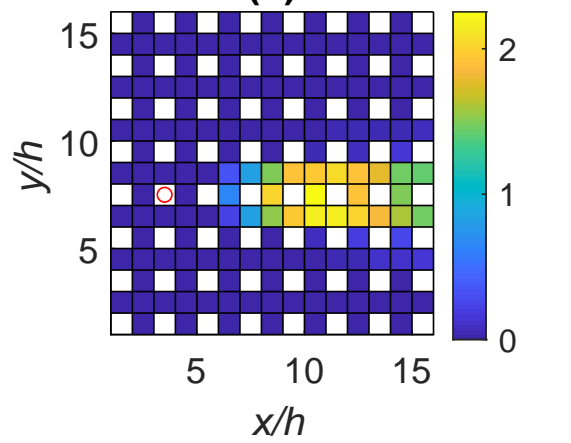

Fig. 6 Magnitude of facet-averaged vertical flux through the canopy top (a) and (b) upward, and (c) and (d) downward, for the ground source (left panels) and elevated source (right panels) for the $0^{\circ}$ run. Locations of ground source at $z=0.0625 h$ and elevated source at $z=2 h$ are denoted by red crosses and circles respectively.

posed into upward and downward components respectively. Fig. 6 shows a map of this upward and downward flux for both sources, with an average value calculated over the top surface of each box at $z=h$. For the ground source there is a disproportionately large upward flux over the source location and in the adjacent channels (Fig. 6a). Hence, there is strong initial detrainment in the immediate vicinity of the source. Upward fluxes decrease monotonically with distance from the source (see also Fig. 7). Re-entrainment is maximum in the very next canyon downwind of the release (Fig. 6c). Thereafter, its magnitude decreases monotonically over subsequent canyons. Re-entrainment fluxes are much lower over the adjacent channels. Interestingly, there is a small amount of downward flux even in the canyon in which the source is located.

For the elevated source, both the upward and downward fluxes span a more extended area. Fig. 6d shows that the downward (entrainment) flux over both canyons and channels increase to a maximum distance of $7 \mathrm{~h}$ downstream of the source before gradually decreasing. This is a consequence of the opposite effects of the plume above the array growing wider to reach the top of the array and diluting in concentration as it continues to grow. Fig $6 \mathrm{~b}$ shows that 

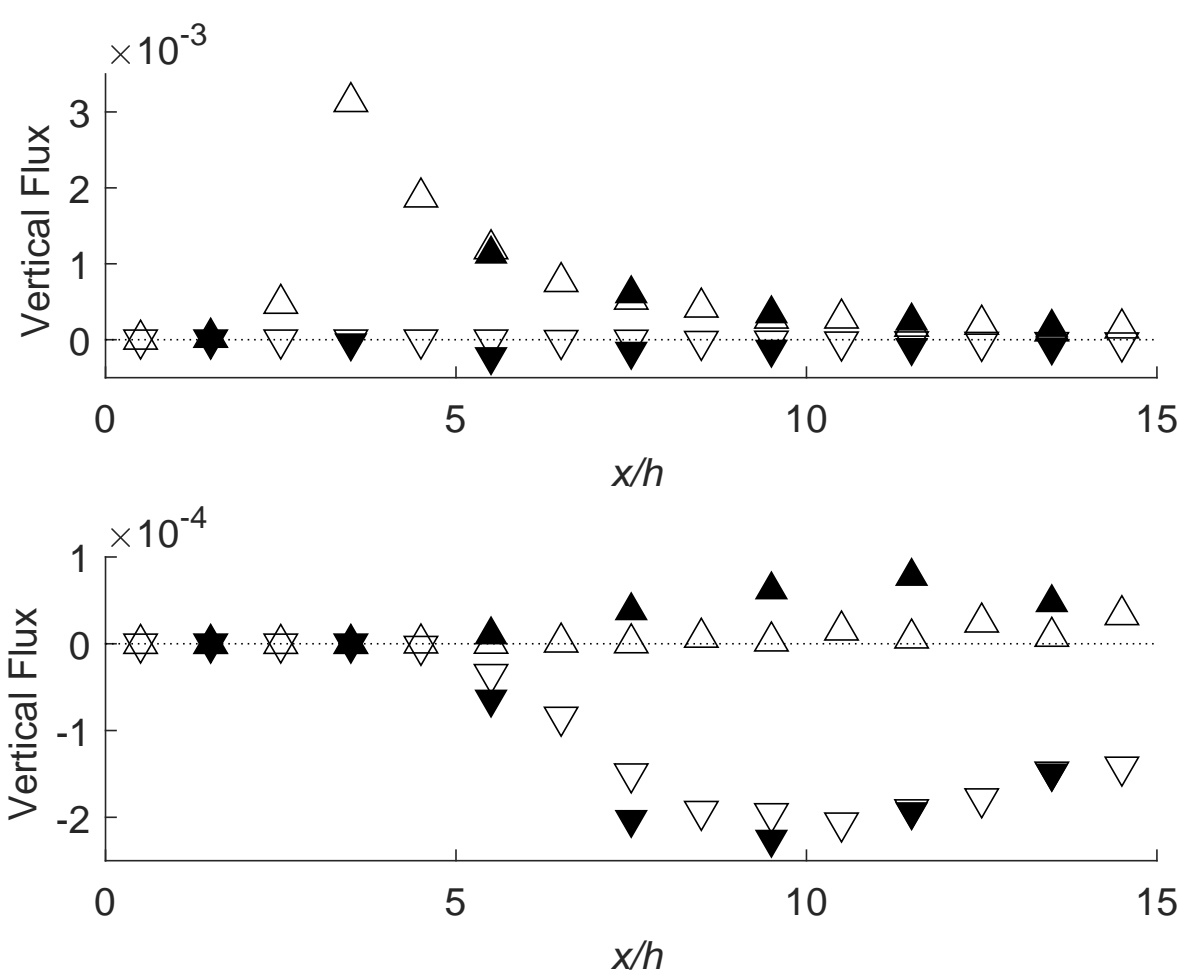

Fig. 7 Facet-averaged vertical flux through the canopy top at different distances along the mean wind direction for (a) the ground source, (b) the elevated source, for the $0^{\circ}$ run. Upward fluxes are denoted by upward-pointing triangles and downward fluxes by downwardpointing triangles. Filled symbols correspond to lateral locations at $y=6.5 h$ and empty symbols at $y=7.5 h$ respectively.

as soon as material has been entrained into the network, some of it is detrained back into the air above. Mirroring the behaviour of the downward flux, the upward flux over canyons first increases to a maximum, then decreases again. The location of the maximum is one canyon downstream of the location of maximum entrainment, at a distance of $9 h$ from the source. The upward flux over channels is small in comparison, and is larger between cubes.

Fig. 7 shows the facet-averaged vertical flux through the canopy top as a function of distance along the mean wind direction at lateral locations $y=6.5 \mathrm{~h}$ and $y=7.5 h$ for the ground source (Fig. 7a) and the elevated source (Fig. 7b). Again, these locations were chosen because most of scalar is channelled along the unobstructed streets adjacent to the source location. The fluxes have been decomposed into an upward component (arrow up) and a downward component (arrow down). Once again the flux due to the initial detrainment from the canyon where the ground source is located (at $z=4 h$ ) is off the scale at 0.014 , compared to the value of 0.0031 in the channel adjacent to it. Thereafter, the upward fluxes from the canyons and the adjacent channels are of a similar magnitude. As already pointed out, the downward (entrainment) flux 

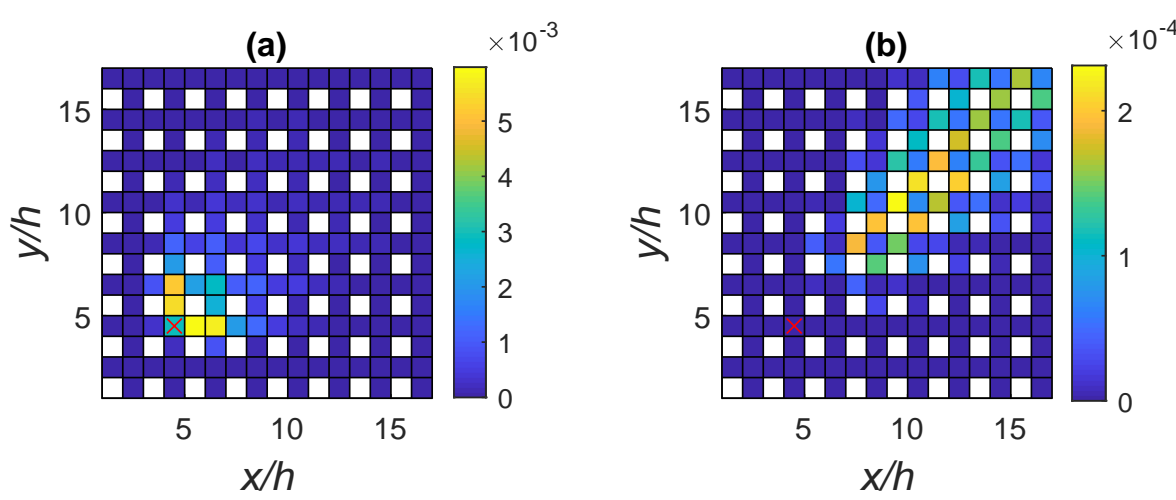

Fig. 8 Magnitude of facet-averaged vertical flux through the canopy top (a) upward, and (b) downward, for the $45^{\circ}$ run. Location of ground source at $z=0.0625 h$ is denoted by a red cross.

is only appreciable over the canyons for this configuration. It has a maximum value over the very next canyon from the source, decreasing monotonically thereafter. For the elevated source, the downward fluxes over the canyons and channels have a comparable magnitude, except close to the source, at $x=6 h$, where the flux over the canyon has a magnitude twice that over the adjacent channel. Thereafter, they rapidly converge until they are virtually identical at $x=12 h$. Overall, the downward flux from the elevated source increases sharply over a distance of $5 h$, peaking at $x=10 h$, then decreases more slowly over a further distance of $5 h$.

The spatial distribution of the main areas of detrainment and re-entrainment for the $45^{\circ}$ run is shown in Fig. 8. They are more laterally extended compared to the $0^{\circ}$ run due to enhanced topological dispersion. The initial wider plume in the canopy produces a more extended detrained plume above which then causes re-entrainment over a wider area downstream. This release scenario appears conducive to the contamination of the widest area among the three cases considered, although the resulting highest concentration levels would be reduced as a result.

\section{Evaluation of Time Scales for Scalar Transport Through the Network}

The results of the previous section indicate that entrainment into the urban street network from a source above it, and re-entrainment of material previously released within it, can both significantly alter the steady-state mean concentration pattern. In this section we investigate the temporal development of the concentration. Given a sudden onset of a release, how quickly does the material propagate, via different processes, through the street network? 


\begin{tabular}{cccc}
\hline DNS run & $U_{w t h}$ & $U_{a b v}$ & $E$ \\
\hline $0^{\circ}$ & 2.9 & 6.8 & 0.25 \\
$45^{\circ}$ & 1.2 & 3.4 & 0.40
\end{tabular}

Table 1 Dimensionless advection and detrainment velocities normalised by $u_{\tau}$.

\subsection{Horizontal Advection and Vertical Exchange Time Scales}

It is instructive to compute typical velocities and time scales associated with individual dispersion processes. This helps to determine which processes control the time evolution of a release. Different choices are possible in defining these velocities and time scales. Following Goulart et al. (2018), we define a facet-averaged advection velocity component $\left\langle\bar{u}_{i}\right\rangle_{k}$ as an area average over a box facet $k$ perpendicular to the component in question. A flux advection velocity is defined as the ratio between the advective flux $\overline{c u_{i}}$ through a street and the volume-averaged mean concentration $\langle\bar{c}\rangle$ within that street. Goulart et al. (2018) also define a vertical detrainment velocity $E$ as the ratio between the vertical turbulent flux and the difference between the volume-averaged concentrations within and immediately above a street. Advection and detrainment velocities calculated according to these definitions and non-dimensionalised by the friction velocity $u_{\tau}$ are given in Table 1 for both DNS runs. The results show that the advection velocities just above the array are roughly 2 to 3 times those within for both wind directions. However, the vertical exchange velocity for the $0^{\circ}$ run is only about a tenth of the advection velocity within the array, whereas the corresponding ratio for the $45^{\circ}$ run is up to a third.

The large difference in the ratio of detrainment to advection velocities for the two wind directions has implications for the dispersion and consequently on the resulting concentrations. For instance, despite the fact that the nominal velocity above the array is larger for the $0^{\circ}$ flow, it will transfer a smaller fraction of any scalar released within the array to above than for a $45^{\circ}$ wind direction. Given the connection of breathability to the vertical exchange velocity (Neophytou and Britter 2005; Panagiotou et al. 2013), the detrainment velocity (which is related to the exchange velocity, though not identical in definition) is a relevant parameter for ventilation. The values in Table 1 show that, even with lower advection velocity the oblique case has higher breathability capacity and is hence more efficient in reducing the concentration within the array. This is consistent with our earlier findings.

An advection and a vertical exchange time scale may be defined as the ratio between a horizontal and vertical characteristic length scale and the advection and detrainment velocity respectively. For the arrays considered here, this characteristic length scale can be taken to be $h$ in both the horizontal and the vertical directions. Values calculated from the DNS data and nondimensionalised by the eddy turnover time $T=h / u_{\tau}$ are given in Table 2 . It is important to point out that these are average values both in time and in space (facet-averaged), and that some variability in actual time scales is inevitable. 


\begin{tabular}{cccc}
\hline DNS run & $T_{w t h}$ & $T_{a b v}$ & $T_{E}$ \\
\hline $0^{\circ}$ & 0.34 & 0.15 & 4.0 \\
$45^{\circ}$ & 0.85 & 0.29 & 2.5
\end{tabular}

Table 2 Dimensionless time scales associated with horizontal advection and vertical exchange.

\subsection{How Long Does It Take for a Localised Release to Reach a Given Street?}

The typical time scales computed in the last section allow simple estimates to be made of the time it takes for a release at a given point to first reach any given street. This time is determined by the quickest pathway linking any two points in the network. We emphasize that this is an inherently transient characteristic that is subject to statistical fluctuations. In any given realization of a sudden release, the initial propagation can take any one of several paths. A large number of such realizations would then be expected to reproduce the probability of traversing these paths - the larger the ensemble, the more representative the results will be. In this section, we make use of the DNS data to estimate the minimum time to reach a given street in the network and interpret the results on the basis of the time scales computed in the last section. In the DNS the scalar release is switched on at a specific time. Hence, it is possible to track the onset and initial growth of the scalar concentration as it propagates through the street network and above. One limitation is the relatively small ensemble size in the DNS - there are 16 releases for the $45^{\circ}$ run, 12 ground source releases for the $0^{\circ}$ run, and only 4 elevated source releases for the $0^{\circ}$ run. Hence, the resulting ensemble averages for the latter are noisier, but still instructive enough for the purpose of providing rough estimates.

Fig. 9 shows a map of the non-dimensional time of first appearance of scalar concentration resulting from a ground source release in the $0^{\circ}$ run, superimposed on the initial part of the time series from which this time is derived. The location of the release is in the top row, middle column. The time of first appearance generally increases monotonically downstream, roughly linearly, consistent with advection being the dominant process. The exceptions are the two columns on the extreme right, close to the source. The very large values in the top right is because it is unlikely for the scalar to reach there. The left-right asymmetry is due to the small puff ensemble size. More generally, it takes much longer for the scalar to propagate laterally than streamwise for this flow direction since topological dispersion is restricted, and lateral turbulent diffusion is also weak (Goulart et al. 2018). Although re-entrainment does occur, particularly over the canyons aligned with the source, it does not significantly alter the time that scalar first appears because the vertical exchange time scale is so much larger than the advection time scale in the canopy.

The corresponding results for the elevated release above the street network are shown in Fig. 10. In this case the source is located above the middle cube in the top row. In contrast to the ground release, entrainment is the predominant process here, and it modifies the times of first appearance and their distribution 


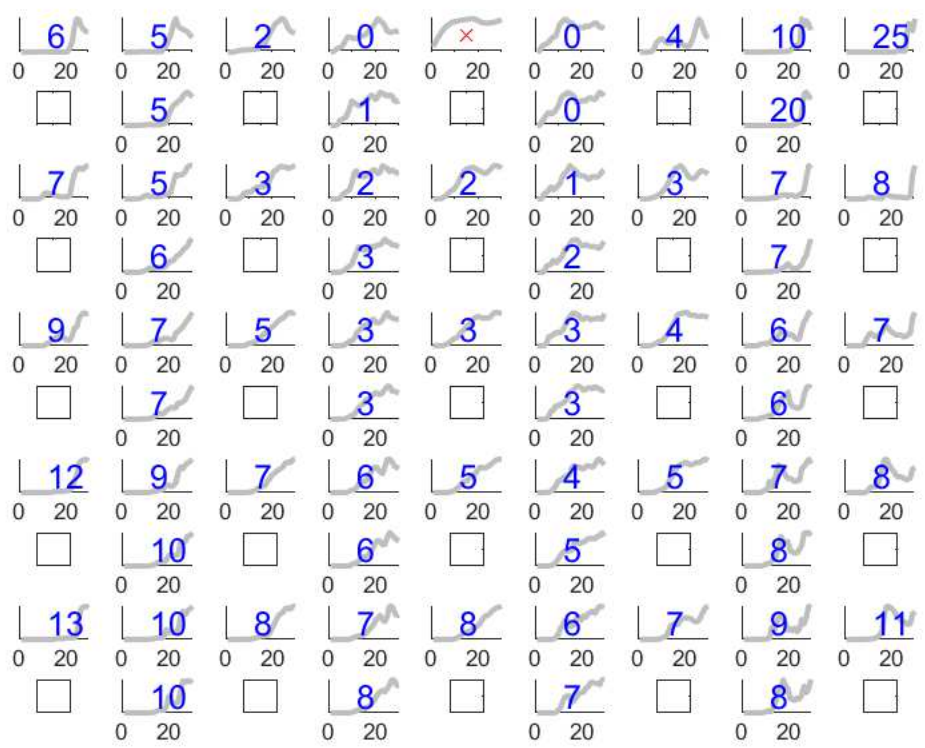

Fig. 9 Map showing non-dimensional time to reach each street for the $0^{\circ}$ run for the ground source located in the middle column of the top row and denoted by a red cross. The horizontal axis has been scaled in units of the non-dimensional time and the vertical axis has been scaled by the maximum concentration in this segment of the local time series.

considerably. First, the values are much smaller than corresponding values in Fig. 9. Propagation by entrainment is quicker than advection through the network because the velocity above the network is much larger than that within. The combination of entrainment and advection has the consequence that monotonic increase of the time of first appearance with distance from the source is not strictly respected - there are several instances where the time in a street is lower than in any other adjacent street, i.e. local mimima exist, which are not found in the case of a ground source. Another noteworthy difference is that the numbers are generally closer together and do not vary too much with distance, particularly in the along-wind direction but also in the lateral direction when compared with the ground-source release case (the exception is in the first rows). Indeed in some instances the same value is observed over several consecutive streets, e.g. the value 4 occurs over five successive streets, and 5 occurs over seven streets.

Fig. 11 shows the distribution of first arrival times for the $45^{\circ}$ run, in which the ground source is located near the ground in the intersection at the topmost left corner. This case is more interesting because advection and re-entrainment both play important roles in determining the time of first arrival in different parts of the network. The pathway that yields the quickest combination de- 


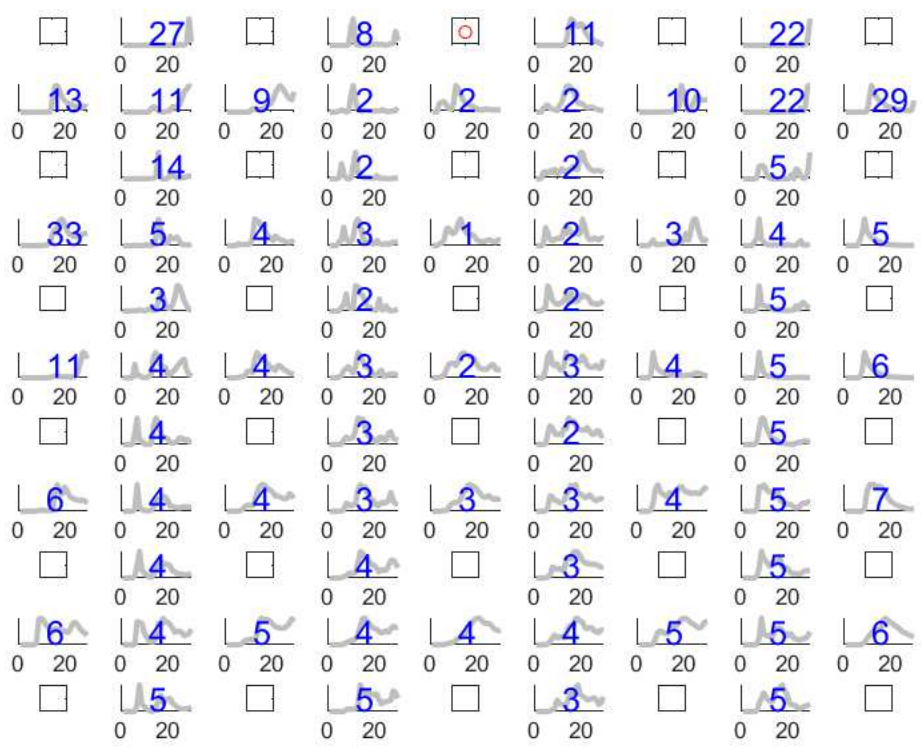

Fig. 10 As in Fig. 9 for the $0^{\circ}$ run for the elevated source located at $z=2 h$ over the middle cube in the top row and denoted by a red circle.

termines this time. In delineating the dispersion pathways, it is important to realise that a so-called 'taxicab' or 'Manhattan' geometry applies within the street network, as opposed to normal Euclidean geometry above. In other words, material being transported within the urban canopy is constrained to follow the rectangular street pattern. Hence, the relevant measure of distance is the so-called Manhattan metric given by $d=|x|+|y|$, in contrast with the straight-line distance $d=\sqrt{x^{2}+y^{2}}$ in the unobstructed space above. This purely geometrical factor on its own slows down scalar transport in comparison with the flow above. The much lower wind speed in the canopy compared to that above is an additional factor with the same effect. The combined effect of these two factors is that the scalar can reach a street downstream faster by first detraining into the air above, followed by advection along the fast flow, then re-entrainment into a downstream street, than by transport through the network alone. Hence, it is common to find smaller values for the time of first arrival at some streets further away than others closer to the source. For example, the intersection at location $(7,7)$ in Fig. 11 registers a non-zero concentration after $t=5$ (in non-dimensional time units), compared to the time of $t=6$ in the street at location $(7,1)$. If transport through the network were the only pathway, then the time to reach $(7,7)$ should have been around twice that to reach $(7,1)$. Hence, re-entrainment provides a shortcut pathway for material to reach further downstream quicker. In an emergency response con- 


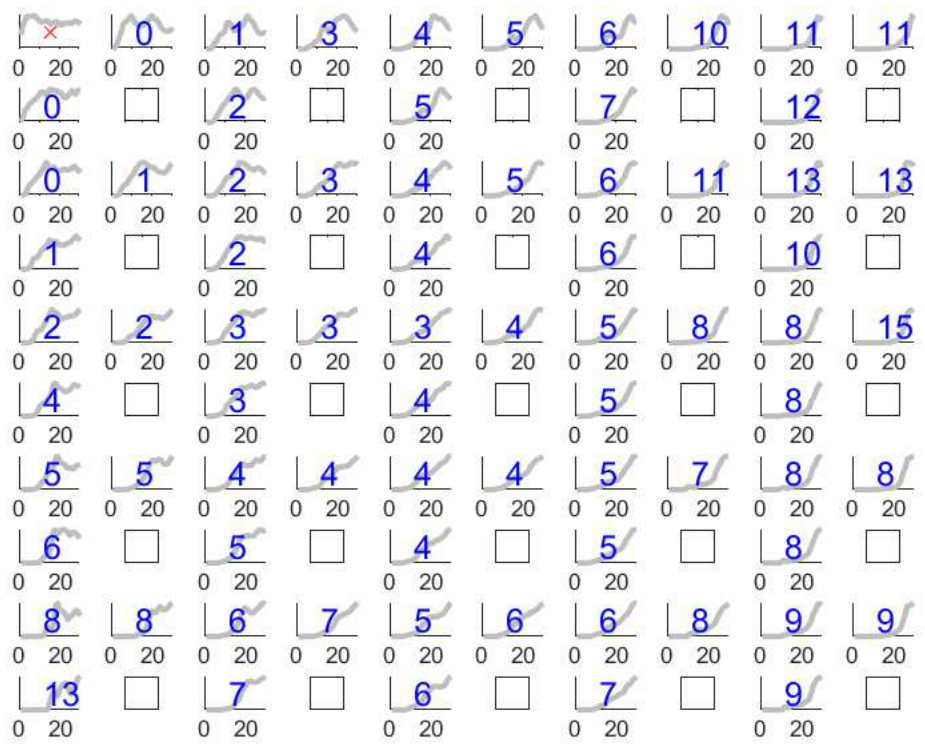

Fig. 11 As in Fig. 9 for the $45^{\circ}$ run for a ground source located in the topmost left hand corner and denoted by a red cross. The mean wind direction is along the leading diagonal.

text, this has implications for which areas need to be attended to or evacuated first.

\section{Conclusions}

In this paper we have investigated the dispersion patterns and propagation from localised sources within and above an idealised street network using DNS data, and related them to underlying dispersion processes in the network. A summary of key results include the following:

- Detailed dispersion patterns are mapped for three different release scenarios: a $0^{\circ}$ (aligned) wind direction with a ground source (GS) and an elevated source (ES); and a $45^{\circ}$ (oblique) wind direction with a ground source. The spatial distribution of mean concentration is related to regions of downward (detrainment) and upward (entrainment) fluxes, which is also mapped. Widening of the in-canopy plume relative to the abovecanopy plume confirms earlier wind-tunnel observations in the literature (DAPPLE 2011).

- Re-entrainment from a GS release occurs just a few streets downstream and a dynamic equilibrium with detrainment is quickly established. This was already known from the earlier studies of Goulart et al. (2018). The new 
result here is that for an ES release entrainment also happens an equally short distance downstream but equilibration with detrainment takes much longer. There are also differences in where it happens quicker (over canyons for GS and over channels for ES).

- There are large differences between the relative magnitudes of advection and detrainment velocities for the aligned and oblique flow directions. This has implications for breathability in a street network, i.e. a change in wind direction can affect ventilation efficiency considerably.

- Characteristic advection and vertical exchange time scales are defined and compared for the different cases considered. These are used to quantify the travel times associated with different dispersion pathways and to interpret the DNS data on times of arrival of the localised release.

- The time of first arrival of the scalar in individual streets in the network is mapped for the three cases. The spatial pattern of this arrival time reflects the relative importance of different dispersion processes in each case:

1. For the aligned flow with a GS advection through the street network is the dominant process and leads to a generally monotonic increase in the time of arrival with distance from the source. Lateral transfer is limited and this is reflected in large arrival times in lateral locations.

2. For the aligned flow with an ES entrainment is, naturally, the dominant process. Times of arrival are much shorter than for the GS because of the faster flow above the network, and are also much more similar. Local mimima in these transit times can occur.

3. For the oblique flow with a GS there is a competition between advection and entrainment. Two factors can give the latter an advantage: the purely geometrical constraint of a taxicab geometry in the street network, coupled with the dynamical effect of reduced advection velocity. This can lead to material reaching remote parts of the network much faster (up to twice as fast in some cases) than it could by advection alone.

Taken together, these results highlight the importance of entrainment and re-entrainment as mechanisms for pollutant spread and dispersion in the urban environment. They show evidence that dispersion pathways and time scales for pollutant transport can be significantly altered as a result of these processes. This has particular implications for emergency-response modelling of toxic releases in urban areas. It can also potentially cast a new perspective on air pollution mitigation strategies, such as in the design of clean air zones and more generally in developing strategies to improve city breathability. Perhaps a key take-home message in this regard is the insight that vertical exchange is a two-way process and that the concept of exchange velocity needs to be augmented in recognition of this fact.

We think the results summarised above and their potential implications are important enough that further studies should be devoted to entrainment in urban canopies. In particular, it would be interesting to pursue a parameter study for different wind directions and array geometries to explore how the 
conclusions change, perhaps using computationally cheaper methods such as large-eddy simulation or Lagrangian stochastic modelling.

Acknowledgements Fernanda Cezana, Elisa V. Goulart and Neyval Costa Reis Jnior gratefully acknowledge funding from National Council for Scientific and Technological Development (CNPq), Capes (Coordination of Superior Level Staff Improvement) - Print Institutional Internationalization Program (88881.311735/2018-01.) and Espirito Santo Research Foundation (FAPES), Brazil. Omduth Coceal gratefully acknowledges funding from the Natural Environment Research Council (NERC) through their National Centre for Atmospheric Science (NCAS) under Grant No. R8/H12/83/002 and from the Engineering and Physical Sciences Research Council for the DIPLOS project (EPSRC Contract No. EP/K040707/1). OC also thanks the Federal University of Espirito Santo (UFES) for funding a two-week visit through their Capes_Print Institutional Internationalization Program.

\section{References}

Belcher SE, Coceal O, Hunt JCR, Carruthers DJ, Robins AG (2012) A review of urban dispersion modelling. Atmospheric Dispersion Modelling Liaison Committee Report, Tech rep

Belcher SE, Coceal O, Goulart EV, Rudd AC, Robins AG (2015) Processes controlling atmospheric dispersion through city centres. J Fluid Mech 763:51-81, DOI https://doi.org/10.1017/jfm.2014.661

Branford S, Coceal O, Thomas TG, Belcher SE (2011) Dispersion of a pointsource release of a passive scalar through an urban-like array for different wind directions. Boundary-Layer Meteorology 139(3):367-394, DOI 10.1007/s10546-011-9589-1

Britter RE, Hanna SR (2003) Flow and dispersion in urban areas. Annual Review of Fluid Mechanics 35(1):469-496, DOI 10.1146/annurev.fluid.35.101101.161147

Carpentieri M, Hayden P, Robins AG (2012) Wind tunnel measurements of pollutant turbulent fluxes in urban intersections. Atmospheric Environment 46:669-674, DOI https://doi.org/10.1016/j.atmosenv.2011.09.083

Carpentieri M, Robins AG, Hayden P, Santi E (2018) Mean and turbulent mass flux measurements in an idealised street network. Environmental Pollution 234:356-367, DOI https://doi.org/10.1016/j.envpol.2017.11.069

Chen L, Hanga J, Sandbergc M, Claessonc L, Sabatinod SD, Wigo $\mathrm{H}$ (2017) The impacts of building height variations and building packing densities on flow adjustment and city breathability in idealized urban models. Building and Environment 118:344-361, DOI https://doi.org/10.1016/j.buildenv.2017.03.042

Coceal O, Thomas TG, Castro IP, Belcher S (2006) Mean flow and turbulence statistics over groups of urban-like cubical obstacles. Bound-Lay Meteorol 121(3):491-519, DOI https://doi.org/10.1007/s10546-006-9076-2

Coceal O, Dobre A, Thomas TG, Belcher SE (2007) Structure of turbulent flow over regular arrays of cubical roughness. Journal of Fluid Mechanics 589:375409, DOI 10.1017/S002211200700794X 
Coceal O, Goulart EV, Branford S, Thomas TG, Belcher SE (2014) Flow structure and near-field dispersion in arrays of building-like obstacles. Journal of Wind Engineering and Industrial Aerodynamics 125:52-68, DOI https://doi.org/10.1016/j.jweia.2013.11.013

DAPPLE (2011) Final reports to the home office; dapple 2009/13 v3. Tech rep

Garbero V, Salizzoni P, Soulhac L (2010) Experimental study of pollutant dispersion within a network of streets. Boundary-Layer Meteorology 136(3):457-487, DOI 10.1007/s10546-010-9511-2

Goulart EV, Coceal O, Belcher SE (2018) Dispersion of a passive scalar within and above an urban street network. Boundary-Layer Meteorology 166(3):351-366, DOI 10.1007/s10546-017-0315-5

Harrison RM (2018) Urban atmospheric chemistry: a very special case for study. npj Climate and Atmospheric Science 1(1):2397-3722, DOI https://doi.org/10.1038/s41612-017-0010-8

Hertwig D, Soulhac L, Fuka V, Auerswald T, Carpentieri M, Hayden P, Robins A, Xie ZT, Coceal O (2018) Evaluation of fast atmospheric dispersion models in a regular street network. Environ Fluid Mech 18(4):1007-1044, DOI 10.1007/s10652-018-9587-7

Moin P, Mahesh K (1998) Direct numerical simulation: A tool in turbulence research. Annual Review of Fluid Mechanics 30(1):539-578, DOI 10.1146/annurev.fluid.30.1.539

Neophytou M, Britter RE (2005) Modelling the wind ow in complex urban topographies: a computational-fluid-dynamics simulation of the central london area. In: Proceedings of the Fifth GRACM International Congress on Computational Mechanics, Limassol, Cyprus

Panagiotou I, Neophytou MKA, Hamlyn D, Britter RE (2013) City breathability as quantified by the exchange velocity and its spatial variation in real inhomogeneous urban geometries: An example from central london urban area. Science of The Total Environment 442:466 - 477, DOI https://doi.org/10.1016/j.scitotenv.2012.09.001

Peng Y, Buccolieri R, Gao Z, Ding W (2020) Indices employed for the assessment of urban outdoor ventilation - A review. Atmospheric Environment 223:955-969, DOI https://doi.org/10.1016/j.atmosenv.2019.117211

Pope SB (2000) Turbulent Flows. Cambridge University Press, DOI $10.1017 /$ CBO9780511840531

Shen Z, Cui G, Zhang Z (2017) Turbulent dispersion of pollutants in urbantype canopies understable stratification conditions. Atmospheric Environment 156:1-14, DOI https://doi.org/10.1016/j.atmosenv.2017.02.017

Soulhac L, Garbero V, Salizzoni P, Mejean P, Perkins R (2009) Flow and dispersion in street intersections. Atmospheric Environment 43(18):29812996, DOI https://doi.org/10.1016/j.atmosenv.2009.02.061

Targa T, Kent A, Stewart R, Coleman P, Bower J, Webster H, Taylor J, Murray V, Mohan R, Aus C (2006) Initial review of air quality aspects of the buncefield oil depot explosion. AEA Technology Environment, Netcen, Tech rep 